\title{
Why Can't Surgeons Treat Older Patients the Same as Younger Patients?
}

\author{
Riccardo A. Audisio ${ }^{1}$ and Charles M. Balch ${ }^{2,3}$ \\ ${ }^{1}$ Department of Surgical Oncology, St Helens Teaching Hospital, University of Liverpool, Liverpool, UK; ${ }^{2}$ Department of \\ Surgical Oncology, University of Texas MD Anderson Cancer Center, Houston, TX; ${ }^{3}$ Division of Surgical Oncology, \\ Department of Surgery, University of Texas Southwestern Medical Center, Dallas, TX
}

Pancreatic cancer is a disease of older people (with a prevalence of only 0.1 per 100,000 in the 20 - to 29 -yearold group and 87.2 per 100,000 after age 80 ), and so is every solid tumor. King and colleagues have published an important article in Annals of Surgical Oncology that highlights our dismal performance as surgeons who undertreat octogenarians who are candidates for pancreatic surgery, highlighting the huge gap of knowledge on such a remarkably important issue. ${ }^{1}$ As a result of undertreatment, cancer-specific survival for older patients is appalling. The authors analyzed data from a series of 431 octogenarians with pancreatic cancer, retrieving clinical information including the Charlson comorbidity index as well as reasons not to treat; they then attempt to correlate treatment planning to outcomes.

Not surprisingly, they confirmed that older cancer patients receive substandard treatment, whether it is surgery for early diseases or chemotherapy for advanced cases. These results are perfectly in line with the literature. In the United Kingdom, we have hard evidence that all elderly patients affected by solid tumors receive less surgery (Fig. 1). ${ }^{2}$

King et al. demonstrate how the deviation from expected treatment guidelines is not related to comorbidities but principally to the patient's chronological age. Consistent with previous reports, the older the patient, the less likely the surgeon is to offer active treatment. For example, surgical resection is performed in over $80 \%$ of pancreatic

\section{(C) Society of Surgical Oncology 2016}

First Received: 11 July 2016;

Published Online: 25 July 2016

R. A. Audisio

e-mail: raudisio@doctors.org.uk cancer patients younger than 70 years, but only $45 \%$ will undergo surgery after age 85 , even if they are fit and present no comorbidities. ${ }^{3}$ Yet with a dismal survival rate for untreated patients, active treatment should be considered and discussed at all times, as it is currently the most powerful factor to improve survival. The report of Riall et al. concluded, "It is important that patients understand the risk of mortality as well as the significant advantage of surgical resection.". 3

Uniquely, King et al. identify the causes for nontreatment options, including contraindication despite a lack of comorbidities and patient refusal. Until differently proven, this is evidence of an inherent bias that older patients are not good candidates for major pancreatic surgery.

In our opinion, age alone is not a contraindication for offering active treatment to older cancer patients; surgical complications primarily correlate to the extent of the operation and comorbidities rather than patient age. ${ }^{4}$ Also, comorbidities are unable to predict surgical outcomes at a population-based level. ${ }^{5}$ It is therefore legitimate to consider the surgeon's experience and judgment as the main contraindications.

Do patients refuse treatment? Patients listen to what we have to say, and they generally take our recommendations under serious consideration. Then they make a decision, often in line with our advice. In the series of King et al., one in three patients refused; but what does this really mean? At multidisciplinary meetings, we hear, "This patient does not want surgery." Of course nobody likes surgery. But have we been able to communicate with the patient plainly and convincingly? Have we explained how surgery is the only potentially curative option? That surgery is feasible even in the older patient? That postoperative pain is usually very well controlled, while not receiving surgery would be a far more painful 


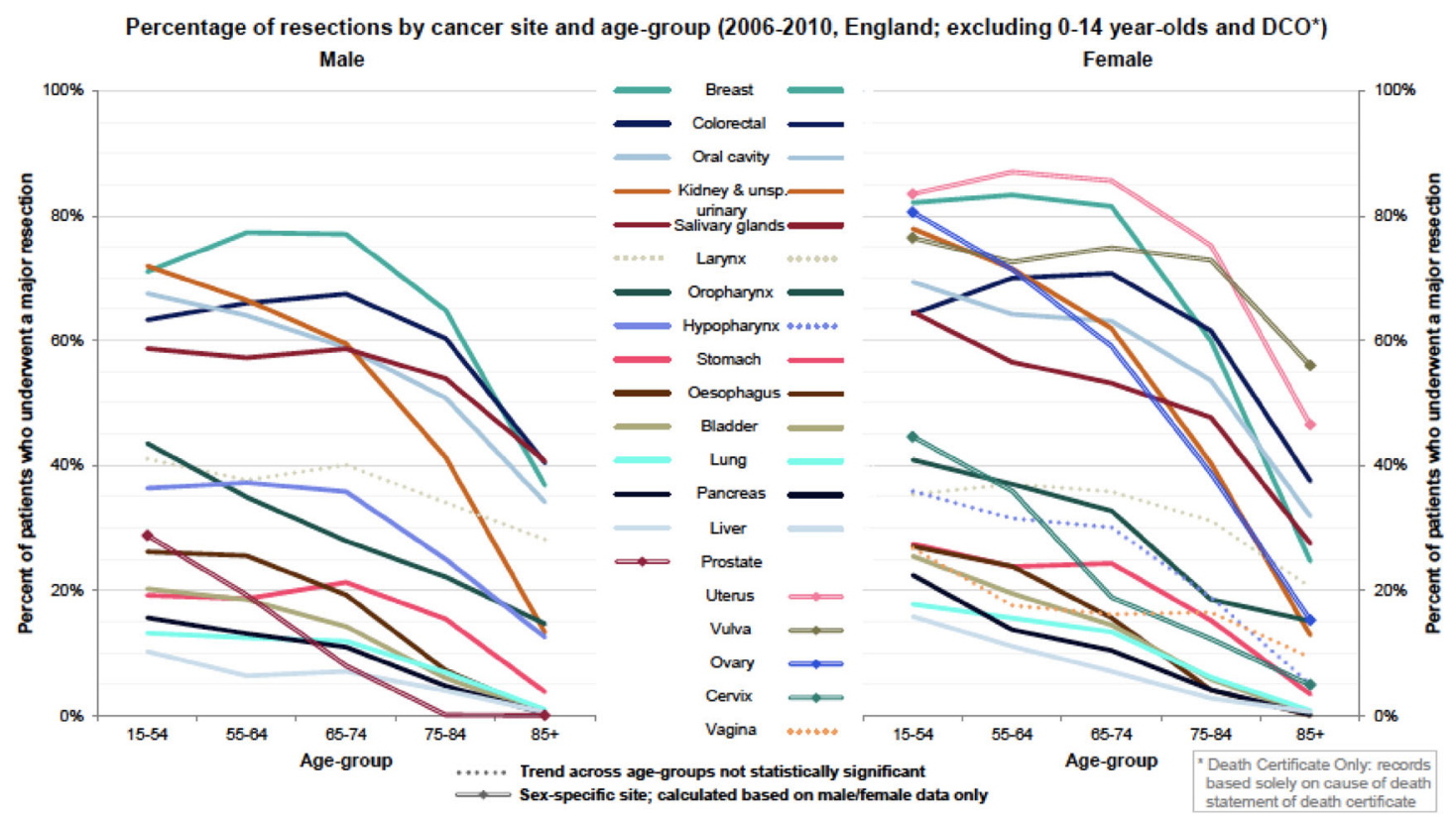

FIG. 1 Major resections by cancer site, England, 2006-2010

alternative? That the operative risks, although present, are considerably reduced, especially in expert hands? That the alternative to surgery is a death sentence within a few months?

While attempting to justify our inclination to understate surgical opinions to our patients, we should consider financial matters. At a time when cancer outcomes are modestly improving while costs are skyrocketing as a result of unaffordable medical treatments, we should be also be aware of the high financial cost of operating on patients who are a poor surgical risk-not only to the patients but to us, the surgeons. Operative mortality, complications, and a lengthy hospital stay will look bad on our records; the poor outcome will be there to prove our poor performance; and financial repercussions are likely to affect our units and hospitals. Medical oncologists are applauded for developing and prescribing unsustainable regimens, while surgical oncologists are penalized for operating on older patients, even if they are reasonably fit.

This is, once again, a desperate call for frailty assessment; the time has come to bring assessment tools into practice. $^{6,7}$ They are inexpensive and feasible. They can predict outcomes and should become part of our basic armamentarium to allow comparisons, to permit informed patient consent, and to provide accurate answers to managerial and health economic issues. ${ }^{8-10}$

Perhaps the time has come to recruit a large series of older cancer patients into a prospective registry, where basic screening tools are recorded preoperatively (Timed Up \& Go test, nutrition, depression, activities of daily living, and instrumental activities of daily living); patients will then be operated on according to standard practice. Short- and long-term reassessments will be performed at 3 and 12 months to better understand which tool (or combination of tools) best predicts surgical outcomes. Such a phase 4 trial is being launched by the Surgical Task Force of the International Society of Surgical Oncology. All interested surgeons are welcome to contribute.

DISCLOSURE The authors declare no conflict of interest.

\section{REFERENCES}

1. King et al. Ann Surg Oncol. In press.

2. National Cancer Intelligence Network. Major resections by cancer site, in England; 2006 to 2010. National Cancer Intelligence Network short report. http://www.ncin.org.uk/about_ncin/major_ resections.

3. Riall TS, Sheffield KM, Kuo YF, Townsend CM Jr, Goodwin JS. Resection benefits older adults with locoregional pancreatic cancer despite greater short-term morbidity and mortality. $J \mathrm{Am}$ Geriatr Soc. 2011;59:647-54.

4. Audisio RA, Gennari R, Sunouchi K, et al. Preoperative assessment of cancer in elderly patients: a pilot study. Support Cancer Ther. 2003;1:55-60.

5. Lemmens VE, Janssen-Heijnen ML, Houterman S, et al. Which comorbid conditions predict complications after surgery for colorectal cancer? World J Surg. 2007;31:192-9.

6. Audisio RA, van Leeuwen BL. Beyond "age": frailty assessment strategies improve care of older patients with cancer. Ann Surg Oncol. 2015;22:3774-5.

7. Audisio RA. Tailoring surgery to elderly patients with cancer. $\mathrm{Br}$ J Surg. 2016;103:e10-1.

8. Huisman MG, Veronese G, Audisio RA, et al; PREOP Study Group. Poor nutritional status is associated with other geriatric domain impairments and adverse postoperative outcomes in 
onco-geriatric surgical patients-a multicentre cohort study. Eur $J$ Surg Oncol. In press.

9. Huisman MG, Audisio RA, Ugolini G, et al. Screening for predictors of adverse outcome in onco-geriatric surgical patients: a multicenter prospective cohort study. Eur J Surg Oncol. 2015;41:844-51.
10. Huisman MG, van Leeuwen BL, Ugolini G, et al. "Timed Up \& Go": a screening tool for predicting 30-day morbidity in oncogeriatric surgical patients? A multicenter cohort study. PLoS One. 2014 24;9:e86863. 